\title{
Estudio Anatómico de la Hiperostosis Porótica en Órbitas Oculares: Cribra Orbitalia en la Población Arqueológica de Tutuquén, Región del Maule, Chile
}

\author{
Anatomical Study of Porotic Hyperostosis in the Eye Orbits: Cribra Orbitalia \\ in the Archaeological Population of Tutuquén, Maule Region, Chile
}

Pérez-Riffo, Marcos ${ }^{1}$; Lindner, Cristian ${ }^{1}$ \& Olave, Enrique ${ }^{2}$

PÉREZ-RIFFO, M.; LINDNER, C. \& OLAVE, E. Estudio anatómico de la hiperostosis porótica en órbitas oculares: Cribra orbitalia en la población arqueológica de Tutuquén, Región del Maule. Chile. Int. J. Morphol., 39(3):721-725, 2021.

RESUMEN: La cribra orbitalia o hiperostosis porótica del techo orbitario es considerada una manifestación ósea microperforativa de condiciones patológicas hematológicas, especialmente la anemia ferropénica. Este hallazgo, se enmarca de manera casi exclusiva a estudios en poblaciones arqueológicas. El propósito de este estudio fue describir la expresión de cribra orbitalia en una población arqueológica de Chile central. Restos óseos de 32 individuos fueron analizados, los cuales fueron obtenidos de la población del Monumento Arqueológico Cementerio Tutuquén, los que están depositados en el Museo Regional de Rancagua. En cada una de la muestras incluidas, se evaluaron períodos de datación, sexo, rango etáreo, presencia v/s ausencia de cribra orbitalia y en los casos en que ésta estuvo presente, se determinó su intensidad mediante visión directa con lente de aumento. Un 59,38 \% de los individuos presentó cribra orbitalia. De éstos, $26,32 \%$ fueron de sexo femenino, 31,58 \% de sexo masculino y 42,10\% indeterminado. De los individuos del período $10.000 \mathrm{AP}, 33,33 \%$ presentó cribra orbitalia; de los individuos del período 7.000 AP un $50 \%$ y de los individuos pertenecientes al período 1.000 AP, un 68,4 \%. Al clasificar la severidad de la cribra orbitalia se observó que 31,57 \% de los individuos presentaron Cribra orbitalia tipo I; 36,84 \% tipo II; 10,52 \% tipo III; 10,52 \% tipo IV y 10,52 \% tipo V. Los datos aportados complementarán el conocimiento morfopatológico de la órbita ocular humana desde el estudio de poblaciones arqueológicas.

PALABRAS CLAVE: Cráneo; Anemia; Cribra orbitalia; Hiperostosis porótica.

\section{INTRODUCCIÓN}

Durante largo tiempo, los cambios anatómicos han sido ampliamente observados en esqueletos de poblaciones antiguas, siendo muchas veces asociados a distintos fenómenos nutricionales, infecciosos o alteraciones hematológicas en la vida del individuo (Stuart-Macadam, 1982; Polo-Cerdá et al., 2001; Galea, 2013; Rivera \& Mirazón Lahr, 2017; Brikley, 2018).

La Cribra orbitalia (CO), corresponde al asentamiento orbitario de la hiperostosis porótica, la cual fue descrita por primera vez por Welcker (1888), quien la definió como una lesión microperforativa que aparece en el techo de la órbita ocular humana, progresando desde lesiones poróticas independientes hasta lesiones trabeculares francamente exofíticas. Este cambio en la anatomía orbitaria es producto de una hipervascularización venosa con hipertrofia e hiperplasia de la médula ósea, cuya existencia también se describe a nivel parietal, femoral y humeral (Nathan \& Hass, 1966; Stuart-Macadam, 1992; Polo-Cerdá et al.; Wapler et al., 2004; Rivera \& Mirazón Lahr).

Si bien la etiopatogenia de la Cribra Orbitalia ha sido ampliamente estudiada, existiendo varias teorías sobre la mesa, cobra un papel especial la hipótesis de la anemia ferropénica como agente etiológico, ya sea por un bajo consumo de hierro en la dieta, aumento de las pérdidas o mecanismos de mal absorción gastrointestinal, lo que estaría tras el origen de estos cambios hiperostósicos en la anatomía ósea (Stuart-Macadam, 1987; Polo-Cerdá et al.; Yildirim et al., 2005; Gómez, 2012; Galea; Brickley, 2018).

El objetivo de este estudio fue describir la expresión de la hiperostosis porótica orbitaria, en una población arqueológica de Chile central, ubicada en el Monumento Ar-

\footnotetext{
${ }^{1}$ Facultad de Medicina, Universidad Católica del Maule, Talca, Chile.

${ }^{2}$ Facultad de Medicina, Universidad de La Frontera, Temuco, Chile.
} 
queológico Cementerio de Tutuquén, cuyos restos óseos pertenecen a un periodo de tiempo que se extiende desde el Holoceno Temprano hasta el Holoceno Tardío, es decir, un lapso temporal aproximado de 10.000 años antes del presente (AP).

\section{MATERIAL Y MÉTODO}

En este estudio, se utilizaron cráneos obtenidos de los restos óseos de la población del Monumento Arqueológico Cementerio Tutuquén. Fueron incluidos según los siguientes criterios: 1 . Haber sido datado y clasificado por expertos como parte de la población del Monumento Arqueológico Cementerio Tutuquén. 2. Limpieza y mantención previa realizada por los técnicos del Laboratorio de Antropología del Consejo de Monumentos Nacionales $(\mathrm{CMN})$, Región del Maule. 3. Preservación parcial o total de al menos una órbita identificable por individuo.

El Monumento Arqueológico Cementerio Tutuquén se ubica en la vertiente occidental de la zona andina meridional sobre el valle longitudinal central entre la Cordillera de la Costa y la Cordillera de los Andes, en la confluencia de los ríos Teno y Lontué, ubicado a 2 kilómetros al oeste de la ciudad de Curicó, Región del Maule, Chile. Corresponde a un lugar que se utilizó como sitio funerario en el período comprendido entre 10.700 AP hasta 800 AP.

La colección arqueológica se ubica actualmente en el Museo Regional de Rancagua. Consta de 42 esqueletos, de los cuales 26 individuos corresponden a adultos y 16 a menores de 18 años. El conjunto de adultos se compone de 16 de sexo masculino y 10 de femenino, mientras que los menores de 18 años están conformados por 5 lactantes $(<2$ años) 8 niños $(2-12$ años) y 3 subadultos $(12-18$ años $)$. La datación, temporalidad y sexo de cada una de las muestras fue registrada por expertos del Monumento Arqueológico Cementerio Tutuquén (Sáez, 2008, 2011).

De los 42 individuos recuperados de las excavaciones arqueológicas, 32 fueron incluidos en esta investigación.

Se identificaron los cráneos de estudio según los criterios de inclusión. De acuerdo al período de hallazgo, se definieron tres períodos de datación: 10.000 AP, 7.000 AP y 1.000 AP. Se agruparon según sexo y estrato etáreo estimado. Se determinó presencia v/s ausencia e intensidad de hiperostosis porótica en los techos orbitarios de cráneos elegibles mediante visión directa con lente de aumento. La severidad de las lesiones poróticas se clasificó según la escala de Stuart-Macadam en los siguientes tipos:
- SM Tipo I: Impresiones óseas similares a capilares (Capillary - like).

- SM Tipo II: Presencia de forámenes finos dispersos.

- SM Tipo III: Presencia de forámenes amplios y aislados.

- SM Tipo IV Presencia de forámenes unidos en estructura trabecular.

- SM Tipo V: Crecimiento exofítico de la estructura trabecular (Stuart-Macadam, 1992; Witkin, 2011).

Los datos fueron tabulados en MS Excel para Windows y analizados con el software IBM SPSS 10.0. Se realizó un análisis descriptivo a través de frecuencias absolutas y relativas expresadas en porcentaje.

\section{RESULTADOS}

Las características generales de la muestra se observan en la Tabla I. Se observó la presencia de hiperostosis porótica orbitaria en 19 casos $(59,38 \%)$. De éstos, 5 (26,32 $\%$ ) fueron de sexo femenino, $6(31,58 \%)$ de sexo masculino y $8(42,10 \%)$ de sexo indeterminado. De la muestra, 10 cráneos $(52,63 \%)$ fueron de individuos adultos, 2 (10,52 $\%)$ de subadultos y $7(36,84 \%)$ de niños.

Tabla I. Características generales de la muestra.

\begin{tabular}{llll}
\hline & & Cráneos (n) & edad (años) \\
\hline \multirow{3}{*}{ Sexo } & Femenino & 11 & $13-50$ \\
& Masculino & 11 & $20-50$ \\
& Indeterminado & 10 & $2-5$ \\
Estrato Etáreo & Niño & 9 & $2-5$ \\
& Subadulto & 3 & $12-15$ \\
& Adulto & 20 & $20-50$ \\
\multirow{5}{*}{ Temporalidad } & $10.000 \mathrm{AP}$ & 3 & $14-15$ \\
& $7.000 \mathrm{AP}$ & 9 & $4,5-50$ \\
\hline Total & $1.000 \mathrm{AP}$ & 20 & $2-50$ \\
\hline
\end{tabular}

La CO se presentó en 1/3 de los individuos examinados del período $10.000 \mathrm{AP}, 5 / 10$ del período $7.000 \mathrm{AP}$ y $13 /$ 19 individuos del período $1.000 \mathrm{AP}$.

Al clasificar la severidad de la hiperostosis en la escala de Stuart-Macadam, se observó que 6 de los 19 cráneos $(31,57 \%)$ presentaron Cribra orbitalia tipo I; 7 (36,84 \%) tipo II; 2 (10,52 \%) tipo III; 2 (10,52\%) tipo IV y $2(10,52$ $\%)$ tipo V (Figs. 1, 2 y 3).

En la Tabla II se expresa la severidad de la hiperostosis porótica orbitaria, con los estratos etáreos y la datación de los cráneos. 
PÉREZ-RIFFO, M.; LINDNER, C. \& OLAVE, E. Estudio anatómico de la hiperostosis porótica en órbitas oculares: Cribra orbitalia en la población arqueológica de Tutuquén, Región del Maule. Chile. Int. J. Morphol., 39(3):721-725, 2021

Tabla II. Clasificación de la severidad de la hiperostosis porótica orbitaria en escala de Stuart-Macadam (1992) (SM) según estrato etáreo y datación de las muestras.

\begin{tabular}{llcccccc}
\hline & & SM Tipo I & SM Tipo II & SM Tipo III & SMTipo IV & SM Tipo V & Total(n) \\
\hline \multirow{3}{*}{ Estrato Etáreo } & Niño & 4 & 3 & 2 & 0 & 1 & 7 \\
& Subadulto & 0 & 1 & 0 & 1 & 0 & 1 \\
& Adulto & 2 & 3 & 0 & 1 & 0 & 10 \\
\multirow{5}{*}{ Datación } & $10.000 \mathrm{AP}$ & 0 & 1 & 0 & 0 & 1 & 1 \\
& $7.000 \mathrm{AP}$ & 2 & 1 & 1 & 0 & 2 & 13 \\
\hline Total (n, \%) & 1.000 AP & 4 & 5 & 1 & 2 & $2(10,52) \%)$ & $19(100 \%)$ \\
\hline
\end{tabular}

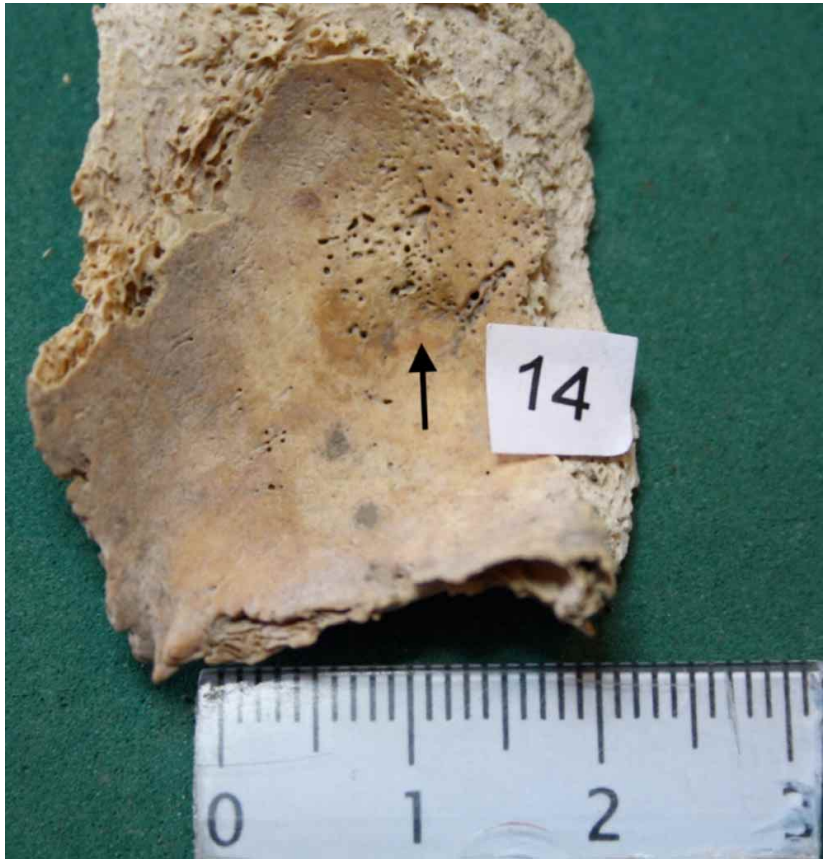

Fig. 1. Cribra orbitalia Stuart-Macadam II en niño de sexo indeterminado. Datación 1.000 AP.

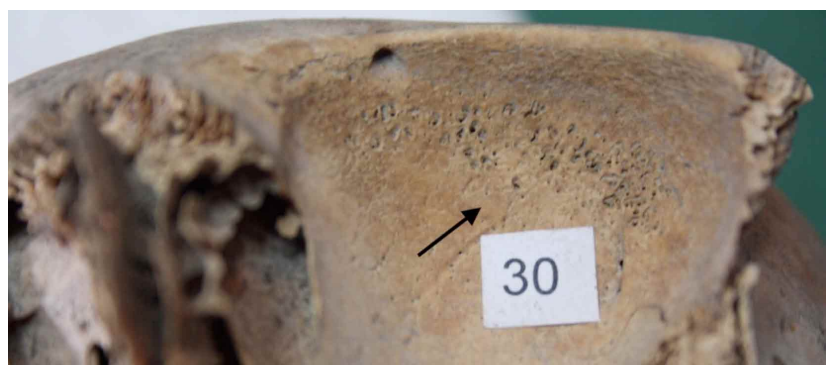

Fig. 2. Cribra orbitalia Stuart-Macadam IV en individuo subadulto femenino. Datación 1.000 AP.

\section{DISCUSIÓN}

La descripción y análisis de distintos indicadores de estrés en el material osteologico humano a partir del estudio anatómico de poblaciones arqueológicas es crucial para com-

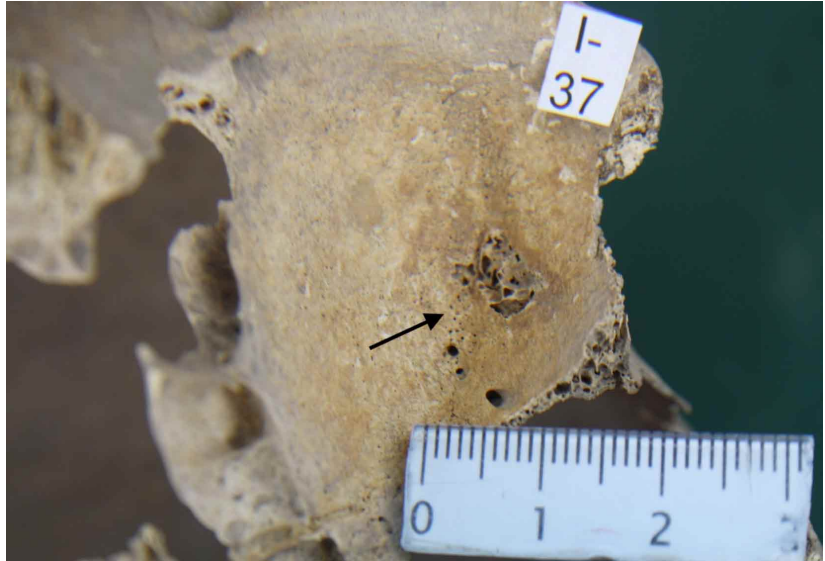

Fig. 3. Cribra orbitalia Stuart-Macadam V en individuo masculino adulto. Datación 1.000 AP.

prender la calidad de vida en el pasado y como sus alteraciones nos afectan a través del tiempo.

Actualmente, se sabe que el hueso reacciona para compensar el estrés ambiental o la falta de nutrición adecuada de una persona. Es por esto, que el estudio de los patrones morfológicos de proliferación y reabsorción ósea nos señalan distintos patrones que actúan como pistas importantes para reconstruir el estilo de vida de una población a partir del análisis y descripción de su anatomía (StuartMacadam, 1982, 1992; Witkin).

Al comparar la presencia de la cribra orbitalia con la literatura, constatamos que la expresión de dicho rasgo en nuestra serie es consistente con distintos reportes internacionales. Witkin y Galea estudiaron los cambios óseos en la colección inglesa de British Royal Infirmary, en la cual destacaba una presencia de hiperostosis porótica orbitaria de 55,96 $\%$ y $53,43 \%$, respectivamente. Por otro lado, Facchini et al. (2004) estudiaron una población romana proveniente de dos necrópolis del nordeste de Italia datadas entre 2000 - 1600 AP, en quienes describieron un $56 \%$ de hiperostosis porótica orbitaria. Salvadei et al. (2001) realizaron su estudio en 140 cráneos obtenidos de dos poblaciones de Italia central (Lucus Feroniae y Selvicciola) datadas entre 1900 y 1300 AP, respec- 
tivamente, mencionando una presencia de $\mathrm{CO}$ total de 53,42 $\%$. Un porcentaje similar reportaron Cornero \& Puche (2002), que informaron una presencia de $53 \%$ de cribra orbitalia en una población arqueológica del centro - este de Argentina datada en 1.760 AP.

Las poblaciones con mayor presencia de $\mathrm{CO}$ provienen de Egipto (1500 AP) y el centro - norte de Polonia (1000 - $500 \mathrm{AP}$ ), en quienes, los autores mencionaron una presencia de $67 \%$ y $85,87 \%$, respectivamente (Fairgrieve \& Molto, 2000; Piontek \& Kozlowski, 2002). En Chile, CostaJunqueira et al. (2000) estudiaron patologías óseas en restos recuperados en una excavación proveniente del morro de Arica (Complejo Cultural Chinchorro), datados entre 3550 - 3220 AP. En ellos, describieron un $25 \%$ de presencia de CO. La menor presencia del rasgo la describe Gómez Mejía (2012) en poblaciones de la sabana de Bogotá. En su estudio, evaluó marcadores óseos de estrés en restos provenientes de Tequendama, Checua, Aguazuque y Vista Hermosa datadas en 11000 AP, 8500 AP, 5025 AP y 3125 AP respectivamente. La $\mathrm{CO}$ resultó ser un hallazgo poco frecuente $(11,68 \%)$, siendo el primer reporte de este rasgo en grupos pre - colombinos de la zona.

En distintos estudios los individuos de sexo femenino poseen la mayor frecuencia de cribra orbitalia (CostaJunqueira et al.; Salvadei et al.; Facchini et al.). Mientras que, si bien en otras poblaciones existe mayor frecuencia de criba en individuos del sexo masculino, los autores coinciden en que la mayor severidad de la cribra se concentró en dos grandes grupos: Individuos de sexo femenino e individuos infantojuveniles,menores de 18 años de edad (Cornero \& Puche; Witkin; Galea).

En nuestro estudio, la presencia de $\mathrm{CO}$ según sexo es similar entre individuos de sexo masculino y femenino, pero se mantiene constante la concentración de las cribras más severas entre los individuos infantojuveniles y aquellos del sexo femenino, coincidiendo con Cornero \& Puche; Salvadei et al.; Piontek \& Kozlowski; Facchini et al.; Witkin y Galea.

Por otra parte, el hecho de que individuos de ambos sexos sufran esta patología, descartaría que el origen del problema sea la pérdida menstrual. Sin embargo, es probable que este fenómeno efectivamente se encuentre asociado a la mayor severidad de las lesiones poróticas en individuos femeninos, comportándose como un factor agravante (Costa-Junqueira et al.; Polo-Cerdá et al.).

Es esperable que los individuos adultos tengan menor prevalencia de cribra orbitalia que aquellos menores de 18 años, ya que, en la adultez, disminuye el requerimiento metabólico propio de los primeros años de desarrollo, con lo que se compensa uno de los principales estímulos de alteración nutricional del fierro y el tejido óseo porotico comienza a recuperar su morfología normal (Facchini et al.; Polo-Cerdá et al.).

A pesar de que existe un gran debate en la literatura sobre la anemia y otras afecciones que puedan relacionarse a las lesiónes hiperostóticas, la observación anteriormente descrita viene a complementar las bases del trabajo experimental realizado por Polo-Cerdá et al., quienes generaron un modelo experimental en 29 ratas Wistar para confirmar la directa relación entre $\mathrm{CO}$ y anemia. En dicho estudio, concluyeron que la presencia de cribra orbitalia es efectivamente un indicador paleonutricional, y que su etiología se basa en tres principales situaciones fisiopatológicas: Aumento de necesidades nutritivas propias de la infancia y juventud, procesos patológicos de malnutrición calórico - protéica, e ingestas dietéticas inadecuadas, concordando con Wapler et al.; Walker et al., 2009 y Brickley.

Los datos aportados por nuestro estudio, complementarán el conocimiento de las manifestaciones morfológicas de variables fisiopatológicas que alteran las condiciones de salud en el ser humano, a través del estudio anatómico de poblaciones arqueológicas.

PÉREZ-RIFFO, M.; LINDNER, C. \& OLAVE, E. Anatomical study of porotic hyperostosis in the eye orbits: Cribra orbitalia in the archaeological population of Tutuquén, Maule Tegion. Chile. Int. J. Morphol., 39(3):721-725, 2021.

SUMMARY: The cribra orbitalia or porotic hyperostosis of the orbital roof is considered a microperforative bone manifestation of hematological pathological conditions, especially iron deficiency anemia. This finding is almost exclusively part of studies in archaeological populations. The purpose of this study was to describe the expression of cribra orbitalia in an archaeological population of central Chile. Bone remains of 32 individuals were analyzed, which were obtained from the population of the Tutuquén Cemetery Archaeological Monument, which are deposited in the Regional Museum of Rancagua. In each of the included samples, dating periods, sex, age range, presence $\mathrm{v} / \mathrm{s}$ absence of cribra orbitalia were evaluated and in the cases in which it was present, its intensity was determined by direct vision with a magnifying lens. The $59.38 \%$ of the individuals presented cribra orbitalia. Of these, $26.32 \%$ were female, $31.58 \%$ male, and $42.10 \%$ undetermined. Of the individuals in the period $10,000 \mathrm{BP}, 33.33 \%$ presented cribra orbitalia; of the individuals of the period 7,000 AP, 50 $\%$ and of the individuals belonging to the period 1,000 AP, $68.4 \%$. When classifying the severity of the cribra orbitalia, it was observed that $31.57 \%$ of the individuals had type I; $36.84 \%$ type II; $10.52 \%$ type III; $10.52 \%$ type IV and $10.52 \%$ type V. The data provided will complement the morpho-pathological knowledge of the human eye orbit from the study of archaeological populations.

KEY WORDS: Skull; Anemia; Cribra orbitalia; Porotic hyperostosis. 


\section{REFERENCIAS BIBLIOGRÁFICAS}

Brickley, M. B. Cribra orbitalia and porotic hyperostosis: A biological approach to diagnosis. Am. J. Phys. Anthropol., 167(4):896-902, 2018.

Cornero, S. \& Puche, R. C. Cribra orbitalia (Porotic hyperostosis) in a prehistoric population of Parana Medio. Medicina (B. Aires), 62(2):16972, 2002.

Costa-Junqueira, M. A.; Cocilovo, J. A. \& Quevedo Kawasaki, S. Patologías óseas, traumas y otros atributos en el grupo arcaico Demorro de Arica, Norte de Chile. Chungara (Arica), 32(1):79-83, 2000

Facchini, F.; Rastelli, E. \& Brasili, P. Cribra orbitalia and cribra cranii in Roman skeletal remains from the Ravenna area and Rimini (I-IV century AD). Int. J. Osteoarchaeol., 14(2):126-36, 2004.

Fairgrieve, S. I. \& Molto, J. E. Cribra orbitalia in two temporally disjunct population samples from the Dakhleh Oasis, Egypt. Am. J. Phys. Anthropol., 111(3):319-31, 2000.

Galea, J. Analysing the Microarchitecture of Cribra Orbitalia Via MicroComputed Tomography in Post-Medieval Remains from the Bristol Royal Infirmary. Tesis de Doctorado. Bristol, University of Bristol, 2013.

Gómez Mejía, J. Análisis de marcadores óseos de estrés en poblaciones del Holoceno Medio y Tardío inicial de la sabana de Bogotá, Colombia. Rev. Colomb. Antropol., 48(1):143-68, 2012.

Nathan, H. \& Haas, N. On the presence of cribra orbitalia in apes and monkeys. Am. J. Phys. Anthropol., 24(3):351-9, 1966.

Piontek, J. \& Kozlowski, T. Frequency of cribra orbitalia in the subadult medieval population from Gruczno, Poland. Int. J. Osteoarchaeol., 12(3):202$8,2002$.

Polo-Cerdá, M.; Miquel-Feucht, M. \& Villalaín-Blanco, J. D. Experimental Cribra Orbitalia in Wistar Rats: An Etiopathogenic Model of Porotic Hyperostosis and other Porotic Phenomena. In: Proceedings of XIIIth European Meeting of the Paleopathology Association, 2001. pp.253-9.

Rivera, F. \& Mirazón Lahr, M. New evidence suggesting a dissociated etiology for cribra orbitalia and porotic hyperostosis. Am. J. Phys. Anthropol., 164(1):76-96, 2017.

Sáez, A. Informe de Laboratorio Bioantropológico de Tutuquén. Manuscrito. Santiago de Chile, Consejo de Monumenots Nacionales, 2008.

Sáez, A. Variabilidad de la morfología cráneofacial en poblaciones humanas holocénicas del área andina meridional. El Cementerio Arqueológico de Tutuquén (7.000-900 AP) Región del Maule, Chile. Estrat. Critic., 5(1):339-51, 2011.

Salvadei, L.; Ricci, F. \& Manzi, G. Porotic hyperostosis as a marker of health and nutritional conditions during childhood: Stud-ies at the transition between imperial Rome and the early middle ages. Am. J. Human Biol., 13(6):709-17, 2001.

Stuart-Macadam, P. A Correlative Study of Paleopathology of the Skull. Tesis de Doctorado. Cambridgde, University of Cambridge, 1982.

Stuart-Macadam, P. Porotic hyperostosis: new evidence to support the anemia theory. Am. J. Phys. Anthropol., 74(4):521-6, 1987.

Stuart-Macadam, P. Porotic hyperostosis: a new perspective. Am. J. Phys. Anthropol., 87(1):39-47, 1992.

Walker, P. L.; Bathurst, R. R.; Richman, R.; Gjerdrum, T. \& Andrushko, V. A. The causes of porotic hyperostosis and cribra orbitalia: a reappraisal of the iron-deficiency-anemia hypothesis. Am. J. Phys. Anthropol., 139(2):109-25, 2009.

Wapler, U.; Crubézy, E. \& Schultz, M. Is cribra orbitalia synonymous with anemia? Analysis and interpretation of cranial pathology in Sudan. Am. J. Phys. Anthropol., 123(4):333-9, 2004.

Welcker, H. Cribra orbitalia, ein etiologisch-diagnostisches Merkmal am Schädel mehrerer Menschenrassen. Arch. Anthropol., 17:1-18, 1888.

Witkin, A. The Health of the Labouring Poor, Surgical and Post-Mortem Procedures at the Bristol Royal Infirmary, 1757 - 1854: A Biohistorical Approach. Tesis de Doctorado. Bristol, University of Bristol, 2011.

Yildirim, T.; Agildere, A. M.; Oguzkurt, L.; Barutcu, O.; Kizilkilic, O.; Kocak, R. \& Niron, E. A. MRI evaluation of cranial bone marrow signal intensity and thickness in chronic anemia. Eur. J. Radiol., 53(1):125-30, 2005.

\author{
Dirección para correspondencia: \\ Marcos Pérez Riffo \\ Universidad Católica del Maule \\ Facultad de Medicina \\ Unidad Anatomía Humana \\ Av. San Miguel \#3605 \\ Talca \\ Región del Maule \\ CHILE
}

E-mail: marcosperezriffo@gmail.com

Recibido : 15-03-2021

Aceptado: 10-04-2021 